\title{
Analyse du site Climat scolaire
}

Jean-Pierre Véran

\section{OpenEdition}

Journals

Édition électronique

URL : http://journals.openedition.org/ries/3986

DOI : $10.4000 /$ ries.3986

ISSN : 2261-4265

\section{Éditeur}

Centre international d'études pédagogiques

\section{Édition imprimée}

Date de publication : 1 septembre 2014

Pagination : 21-22

ISBN : 978-2-85420-604-3

ISSN : 1254-4590

\section{Référence électronique}

Jean-Pierre Véran, «Analyse du site Climat scolaire », Revue internationale d'éducation de Sèvres [En ligne], 66 | septembre 2014, mis en ligne le 01 septembre 2014, consulté le 22 septembre 2020. URL http://journals.openedition.org/ries/3986 ; DOI : https://doi.org/10.4000/ries.3986

Ce document a été généré automatiquement le 22 septembre 2020

(c) Tous droits réservés 


\title{
Analyse du site Climat scolaire
}

\author{
Jean-Pierre Véran
}

\section{NOTE DE L'ÉDITEUR}

Cette note de lecture porte sur un site internet collaboratif : Climat scolaire, accessible à l'adresse suivante : http://www.cndp.fr/climatscolaire/accueil.html.

1 On relève quatre verbes d'action dès la page d'accueil de ce site web collaboratif du ministère français de l'éducation nationale $\mathrm{CANOPE}^{1}$ : comprendre, diagnostiquer, agir et contribuer. L'affirmation que «ce site n'existera pas sans vous » souligne qu'il s'agit d'un site collaboratif, avec un cinquième élément déterminant : les ressources.

Le propos est clair: le climat scolaire est ici envisagé dans le cadre d'une approche systémique, ouvrant sur différents champs d'action : non pas seulement la prévention des violences, mais aussi la qualité de vie à l'école, la justice scolaire, la coéducation, la coopération, les pratiques partenariales, la stratégie d'équipe.

Plusieurs entrées ouvrent sur une fiche de référence, une liste de ressources et les thèmes associés. Les ressources proposent des outils professionnels (par exemple, « interroger mon rôle en tant que chef d'établissement dans la stratégie d'équipe »), des ressources nationales (textes officiels, paroles d'experts, etc.) et académiques (questionnaires, aide au diagnostic, etc.). La fiche de référence est structurée par trois verbes d'action: s'informer (résultats de la recherche et des enquêtes, notamment), agir (en termes d'objectifs stratégiques, opérationnels et d'actions possibles), s'inspirer (à partir d'articles récents ou plus anciens). La fiche se clôt avec des ressources complémentaires.

4 On apprécie ici la valeur ajoutée du web : quelle que soit la porte d'entrée choisie par le lecteur, il accède facilement à la totalité de ce qui concerne sa quête sur le site, sur différents supports, vidéo notamment.

5 Doit être également saluée la variété des ressources proposées, de la circulaire nationale à l'expérimentation locale, d'un rapport d'étude à un outil de repérage académique. L'entrée par les ressources permet de sélectionner soit un des champs 
d'action caractérisant l'approche systémique du climat scolaire, soit tous les champs d'action. Elle permet aussi de choisir la localisation (départementale, académique) ou toutes les localisations. On pourra s'étonner que les ressources produites dans d'autres pays, francophones ou non, ne soient pas proposées, alors qu'elles sont mobilisées à bon escient dans les parties "s'informer » et "s'inspirer » des fiches de référence. Par exemple, dans l'entrée coopération, les fiches " Agir pour briser la solitude en classe » et "Interroger mon rôle en tant que chef d'établissement dans la stratégie d'équipe " font référence aux travaux de Debarbieux et Fotinos, mais aussi à ceux de Rabinowitz et Hall (1977), Osborne (2004), ou Louise Lafortune au Québec (2005). On est surpris également que, par exemple, les travaux hispanophones sur la convivencia escolar n'apparaissent pas où on les attendrait, sur le thème de la convivialité scolaire.

De même, les fiches d'auto-positionnement par métier mériteraient d'être enrichies, en prenant en compte le rôle déterminant et spécifique de certains acteurs. On pense notamment au rôle particulier du professeur-documentaliste, qui accueille les élèves non pas exclusivement en division dans une salle de classe, comme les autres enseignants, mais dans la diversité du public scolaire en un espace spécifique. Dans cet espace de lecture, de recherche, de travail personnel ou collaboratif, l'attention portée à la qualité de l'accueil et du climat est essentielle, comme l'est également l'attention portée aux ressources mises à disposition de tous. On pense aussi au rôle déterminant des assistants d'éducation, en charge de l'encadrement de proximité des élèves dans des espaces stratégiques : cour de récréation, espaces collectifs de rassemblement et de circulation, restaurant scolaire, salles d'étude. Quand on observe que des collégiens interrogés sur la qualité du climat dans l'établissement accordent la plus mauvaise note à la salle d'étude, il y a en effet de quoi aider à l'auto-positionnement des assistants d'éducation et favoriser ainsi leur formation. Or ces personnels ne sont évoqués dans la fiche concernant le conseiller principal d'éducation qu'en deux occasions : «Je forme les personnels de la vie scolaire aux gestes et postures ", " Je veille à la cohérence et à la cohésion de l'équipe en organisant des réunions de service intégrant la politique d'établissement $»$.

7 Il faut sans doute se garder de faire de ces constats un défaut du site, qui a été mis en ligne en mars 2014 et est en plein développement. L'annuaire du réseau des contributeurs est prometteur. Outre le ministère, les académies et leurs cellules climat scolaire, le réseau CANOPE, les associations sont bien représentées, avec les associations d'éducation périscolaire et populaire et d'autres plus locales.

8 On voit, à travers ce site collaboratif, la transformation apportée par les médias numériques au pilotage politique en éducation : les circulaires et textes officiels sont un cadre, mais les travaux de recherche, les expérimentations de terrain, les outils élaborés ici et là sont mis à la disposition de tous, pour que chaque membre et chaque équipe d'établissement scolaire puisse s'informer, enrichir sa culture personnelle sur le sujet, faire le point sur sa contribution à la bonne qualité du climat scolaire, enrichir le site de ressources nouvelles. De cette manière, on peut penser que l'élaboration de la politique pédagogique et éducative de chaque unité d'enseignement prendra en compte un facteur déterminant de la réussite de cette politique : le climat scolaire. 


\section{NOTES}

1. Le bouleversement numérique concerne tout autant la publication et l'édition que l'éducation. C'est pourquoi nous proposons une note de lecture, rédigée en mai 2014, portant sur ce site collaboratif du ministère de l'éducation nationale français et du réseau CANOPÉ, consacré au climat scolaire.

\section{AUTEUR}

\section{JEAN-PIERRE VÉRAN}

Jean-Pierre Véran est inspecteur d'académie honoraire, formateur associé à l'Université Montpellier 2 où il enseigne notamment les politiques éducatives et la gouvernance des organisations scolaires. Il est co-auteur de Le Conseiller principal d'éducation : de la vie scolaire à la politique éducative (2e édition, 2012) et de De l'emploi du temps aux emplois des temps : pour une approche globale du temps scolaire (2011), Berger-Levrault. 